\title{
Conditioned defensive burying in rats free to escape
}

\author{
JOHN P. J. PINEL, DALLAS TREIT, FERIAL LADAK, and A. J. MacLENNAN \\ University of British Columbia, Vancouver, British Columbia V6T 1W5, Canada
}

\begin{abstract}
Rats shocked once by a stationary, wire-wrapped prod bury it if suitable materials are available. Does this conditioned defensive burying occur when rats have the opportunity to flee from the source of aversive stimulation, or is it limited to situations such as those in which it had previously been studied-those in which the relatively small test chamber confined each rat to the immediate vicinity of the prod? In the present experiments, the capacity of rats to flee from the shock prod was enhanced by increasing the floor dimensions of the test chamber up to $200 \times 80 \mathrm{~cm}$ (Experiment 1) or by providing the rats with an opportunity to seek refuge in a separate, safe compartment (Experiment 2). Although both of these modifications to the usual conditioned-defensive-burying paradigm significantly reduced the duration of burying and the height of the accumulated mounds, burying remained well above control levels in all experimental conditions.
\end{abstract}

There are two features shared by most traditional aversive conditioning chambers that can constrain a rat's ability to cope with aversive stimulation. First, in most conventional aversive conditioning environments, there are few, if any, objects that a rat can incorporate into a defensive response; unlike more natural settings, most test chambers have floors constructed of fixed, metal rods through which feces and other materials pass. Second, the exact source of the noxious agent is almost always well disguised; the aversive stimulus is typically a diffuse electric shock administered through a poorly defined source, the grid floor.

Pinel and Treit (1978) recently reported the results of a series of aversive conditioning experiments in which these two features of conventional test chambers were modified. The floor of the chamber was a layer of commercial bedding material rather than the usual grid, and the single shock administered to each experimental subject came from a well-defined source, a small wire-wrapped prod fixed to the wall of the chamber. In this less constraining situation, the behavior of the shocked rats was remarkably different from that usually observed during the course of more traditional aversive conditioning experiments. After the initial reflexive withdrawal, almost every rat sprayed the prod with bedding material from the floor of the chamber, and rats not tested until 20 days after the shock still displayed significant amounts of this burying behavior.

Terlecki, Pinel, and Treit (1979) termed the burial

This research was supported by a National Research Council of Canada grant awarded to John Pinel. Requests for reprints should be sent to John Pinel, Department of Psychology, University of British Columbia, Vancouver, British Columbia V6T IW5, Canada. of sources of aversive stimulation conditioned defensive burying, and they offered three lines of evidence to support the view that the burial of shock prods is guided by the conditioned association of prod and shock. (1) When rats in the test chamber encountered a prod through which they had been shocked in their home cages, they buried it, whereas those previously shocked through a grid did not (Pinel \& Treit, 1978). (2) Changing either the brightness (white or black) or position (front or back of the chamber) of the prod in a 1-min interval between the shock and the test produced systematic reductions in the amount of burying (Pinel, Treit, \& Wilkie, Note 1). (3) Rats shocked by one of two identical prods mounted on opposite walls of the test chamber buried only the shock prod (Pinel \& Treit, 1978). Because defensive burying can also occur as an unconditioned component of the rat's neophobic reaction, it is important to make the distinction between conditioned and unconditioned burying. Terlecki et al. (1979) found that rats entered their experimental environment with an already established tendency to bury some novel objects (unconditioned burying) but that they readily learned to bury any object that had been the source of an aversive stimulation (conditioned defensive burying).

Although investigations of conditioned defensive burying can serve as a basis for criticizing the restrictive methodology of traditional aversive conditioning paradigms, the burying paradigm itself is restrictive in one important way: the ability of subjects to flee from the source of aversive stimulation is always restricted by the boundaries of the conditioning chamber. Previous studies of conditioned defensive burying had all been conducted in relatively small $(44 \times 30 \mathrm{~cm})$ test chambers that did not afford 
the subjects an opportunity even to leave the immediate vicinity of the shock prod. As a result, an important question remained concerning the generality of the conditioned burying response. Is conditioned burying a defensive reaction that occurs only in those cases in which rats have no opportunity for flight? The following experiments were both studies of conditioned defensive burying in situations in which flight from the shock source was a more viable defensive option; the capacity of the rats to flee from the shock prod was increased by increasing the size of the experimental chamber (Experiment 1) or by providing the rats with an opportunity to flee to a separate, safe compartment (Experiment 2).

\section{EXPERIMENT 1}

\section{Method}

The subjects were 100 naive, male, hooded rats (Canadian Breeding Farm and Laboratories, St. Constant, Quebec), weighing between 200 and $250 \mathrm{~g}$. They were housed in groups of 5 or 6 on a floor of a homogeneous commercial bedding material composed of ground corncob (San-i-cel, Paxton Processing Co., Paxton, 111.) in polyethylene cages $(50 \times 40 \times 20 \mathrm{~cm})$, where water and Purina laboratory pellets were continuously available.

The subjects were divided randomly into four groups that were tested in one of four chambers, each $24 \mathrm{~cm}$ in height but with different floor dimensions: small $(25 \times 20 \mathrm{~cm})$, medium $(50 \times 40 \mathrm{~cm})$, large $(100 \times 60 \mathrm{~cm})$, or very large $(200 \times 80 \mathrm{~cm})$. Each chamber had a Plexiglas lid, stained wooden walls, and a floor covered evenly with $5 \mathrm{~cm}$ of the commercial bedding (San-i-cel). The rats in each of the four conditions were placed in groups of about 10 in their respective chambers for three 30 -min habituation periods over the first 3 days of the experiment, and they were placed in the apparatus individually for 3-min periods on each of Days 4 to 7 , inclusive.

Before testing, the rats in each of the three conditions were subdivided randomly into shock and no-shock groups. Thus, the design was essentially a 2 by 4 factorial, with separate groups of shock and no-shock subjects being tested in each of four chambers. Testing was scheduled over Days 5 to 8 , inclusive, in such a way that the proportion of subjects from each of the eight groups was comparable on all 4 of the test days. Following habituation, but before testing, a $5 \times .5 \times .5 \mathrm{~cm}$ wooden prod was fixed to one corner of the chamber, $2 \mathrm{~cm}$ above the bedding material, horizontal to the floor, and at a 45-deg angle from each of the adjacent walls. To begin the conditioning trial, each shock subject was placed in the chamber with its back to the prod, approximately $30 \mathrm{~cm}$ away. When each of the shock rats touched the prod, a brief shock, initiated by the experimenter and terminated by the withdrawal of the subject, was delivered between two uninsulated wires wrapped around the prod. The shock circuit, comprising an $800-\mathrm{V}$ current source and an $80-\mathrm{k} \Omega$ dropping resistor, delivers shocks averaging $7.9 \mathrm{~mA}(\mathrm{SD}=1.47 \mathrm{~mA})$ in intensity and $42.9 \mathrm{msec}$ $(\mathrm{SD}=9.8 \mathrm{msec}$ ) in duration when used in this fashion (Pinel \& Treit, 1978). The subjects in the four no-shock groups were treated identically to the shock subjects except that they were not shocked. All subjects remained in the chamber for $15 \mathrm{~min}$ following the initial prod contact, and their behavior was observed during this period over closed-circuit television.

The burying behavior of rats in this situation typically consists of a series of stereotyped sequences that begin with the rat facing the prod from a distant part of the apparatus (Pinel \& Treit, 1978). The rat moves toward the prod, pushing and spraying a pile of bedding material at the prod with rapid movements of the snout and forepaws. The invariant components of this behavioral pattern are the shoveling movements of the snout and the alter- nating pushing motions of the forelimbs by which the rat displaces material toward the prod. Thus, it was the durations of these directed bursts of snout and forelimb spraying that were recorded. The interobserver reliability of the burying duration measure was established by a separate pilot study in which two observers independently recorded the duration of prod burying by 10 shocked rats during 15 -min tests. The product-moment correlation between the two sets of scores was .988 . In order to corroborate the behavioral measures in the present study, the height of the bedding material at the base of the prod was measured after each test.

In anticipation of the possibility that subjects conditioned and tested in the larger boxes might be difficult to shock in a reasonable period of time, more subjects were assigned to those groups; however, only 2 of the 100 subjects failed to contact the prod within the prescribed 15 -min time limit. From smallest to largest, the number of experimental subjects completing the experiment in each of the four chambers was $12,14,14$, and 14, compared with $10,10,12$, and 12 in the respective control groups.

\section{Results and Discussion}

It is readily apparent from Figure 1 that the shock rats spent considerably more time burying the prod than did their respective no-shock controls, regardless of the size of the chamber. In fact, although 45 of the 54 subjects in the four shock conditions sprayed at least some bedding at the prod, this behavior was observed in only 9 of the 44 no-shock control subjects. The significance of the difference in the duration of burying by shock and no-shock subjects was confirmed by an examination of the appropriate main effect in a 2 by 4 analysis of variance $[F(1,90)$ $=54.07, \mathrm{p}<.0001]$. Planned orthogonal comparisons revealed that the shock rats spent significantly more time burying than did their respective no-shock controls in each of the four chambers [small, $t(21)$ $=5.49, \mathrm{p}<.0003$; medium, $\mathrm{t}(23)=3.55, \mathrm{p}<.003$;

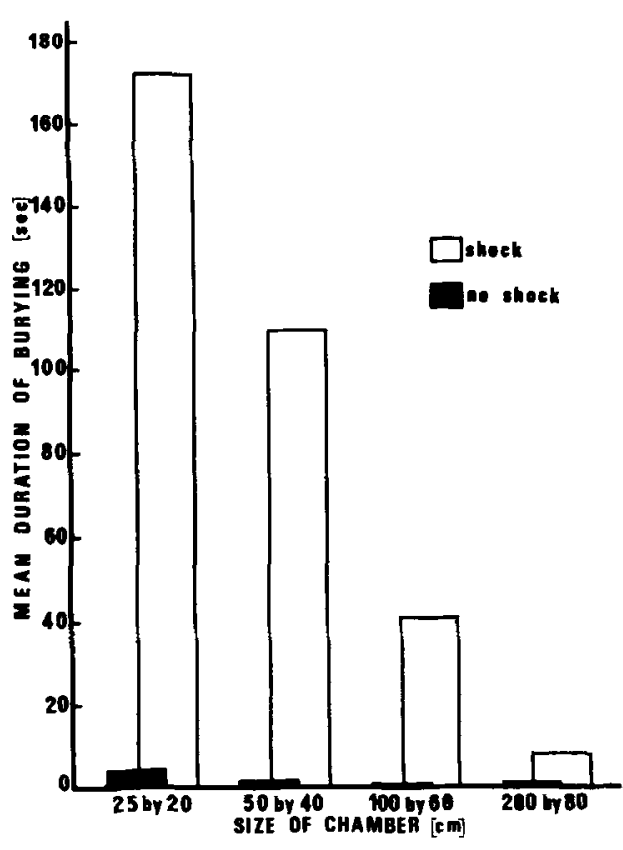

Figure 1. Mean duration of burying by shock and no-shock subjects tested in each of the four chambers. 
large, $t(25)=3.29, p<.004$; very large, $t(25)=2.27$, $\mathrm{p}<.05]$.

It is also evident from Figure 1 that the duration of burying in the shock rats decreased as the size of the chamber increased. The significance of this effect was confirmed by an examination of the main effect of chamber size on the duration of burying $[\mathrm{F}(3,90)=15.07, \mathrm{p}<.0001]$. Because chamber size had its major effect on the animals in the shock groups, the interaction effect was also significant $[F(3,90)=11.45, p<.0001]$. The groups did not differ significantly in their latencies to make prod contact during the conditioning trial $[F(7,90)=1.17$, $\mathrm{p}>.20]$.

These behavioral results were confirmed by comparable analyses of the height data (see Figure 2). Shock subjects as a group accumulated significantly higher piles of bedding at the prod than did no-shock subjects $[F(1,90)=79.58, p<.0001]$; subjects tested in smaller chambers accumulated significantly higher piles $[F(3,90)=20.44, p<.0001]$; and the interaction effect was again significant $[F(3,90)=8.97, p<.0001]$. Planned orthogonal comparisons revealed that the shock rats accumulated significantly higher piles of bedding at the prod than did their respective noshock controls in each of the four chambers [small, $\mathrm{t}(21)=25.45, \mathrm{p}<.00001 ;$ medium, $\mathrm{t}(23)=3.33$, $\mathrm{p}<.0003$; large, $\mathrm{t}(25)=2.93, \mathrm{p}<.0008$; very large, $\mathrm{t}(25)=3.48, \mathrm{p}<.003$ ]. A few of the no-shock subjects dug into the bedding just under the prod, thus reducing the average height of the bedding at the prod below the original level of $5 \mathrm{~cm}$ (see Figure 2).

The topography of the burying response was comparable to that described by Pinel and Treit (1978); the shock rats pushed and sprayed the bedding mate-

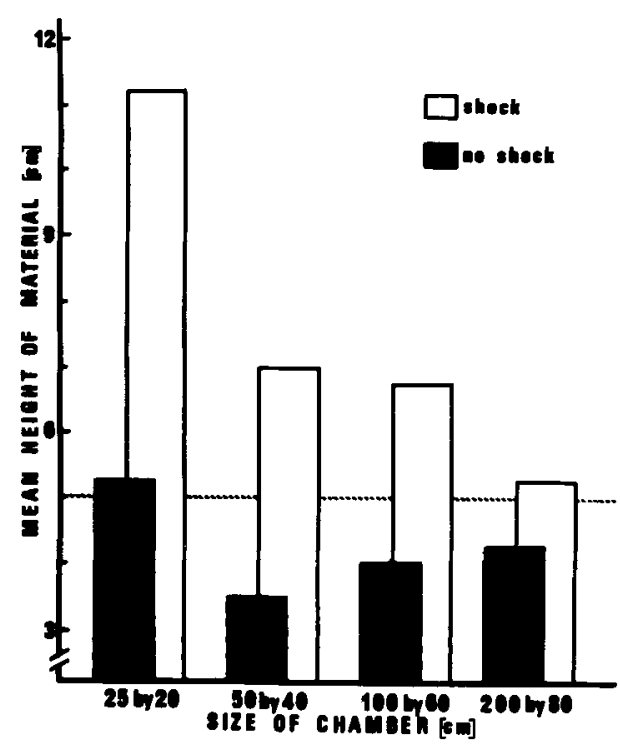

Figure 2. Mean height of bedding material at the prod accumulated by shock and no-shock subjects tested in each of the four chambers. rial with their snouts and forepaws as they moved toward the prod. This pattern varied in one minor respect as a function of chamber size. Some rats tested in the larger chambers tended to spray the bedding material from a distance, and thus, in some instances, the accumulated bedding material was not always centered directly over the prod. Some of these rats accumulated a pile of bedding in front of the prod. Therefore, the height of the pile of bedding material accumulated directly over the prod is not always a sensitive measure of burying behavior in large chambers.

Thus, increases in the floor dimensions of the test chamber up to $200 \times 80 \mathrm{~cm}, 12$ times the floor area of the apparatus used by Pinel and Treit (1978), were not sufficient to eliminate the conditioned defensive burying of a well-defined shock source. Clearly, relatively small test chambers are not a necessary condition for conditioned defensive burying. However, increases in the size of the chamber did reduce both the amount of time that the shock subjects spent spraying bedding and the height of the piles that they accumulated at the prod.

\section{EXPERIMENT 2}

In Experiment 1, the opportunity for rats to flee from a well-defined source of aversive stimulation was increased by enlarging the experimental chamber. Although the general purpose of Experiment 2 was the same as that of Experiment 1, that is, to determine whether rats given the opportunity to flee from a source of painful electric shock would bury it, the approach to the problem was different. In Experiment 2, the rats were tested in an apparatus with two interconnected chambers, thus providing the rats with an opportunity to flee to a "safe" compartment. Do rats shocked by a well-defined object in one chamber simply flee to the safe chamber or do they remain to bury the shock source?

\footnotetext{
Method

The 64250 - to $300-\mathrm{g}$ rats serving as subjects were purchased, housed, and maintained as in Experiment 1 . The apparatus was a $50 \times 40 \times 24 \mathrm{~cm}$ box that for half of the subjects was divided into two $40 \times 25 \mathrm{~cm}$ compartments by a divider with a 10-cm gap in the center. Half of the rats $(n=32)$ were habituated and tested with the divider in place; the others were habituated and tested without it. The floor of the apparatus was covered with $5 \mathrm{~cm}$ of San-i-cel bedding material during all phases of the experiment. Habituation occurred, as in Experiment 1, over a 6-day period. On Days 1 to 3, inclusive, the rats were habituated to the apparatus for $30-\mathrm{min}$ periods in groups of five or six, whereas, on Days 4 to 6 , inclu sive, they were habituated individually for $3 \mathrm{~min}$ each day.

Prior to conditioning and testing, the rats in the one-compartment and two-compartment conditions were assigned randomly to shock and no-shock conditions to form four groups of 16 subjects each. Thus, the design was a 2 by 2 factorial.

The subjects were shocked and tested in accordance with the methods of Experiment 1 . On the test day, each of the 32 shock subjects (16 one-compartment and 16 iwo-compartment rats) was
} 
placed in the chamber $10 \mathrm{~cm}$ from the prod that had been mounted $2 \mathrm{~cm}$ above the bedding in the center of one end wall. All subjects were positioned so that they faced away from the prod toward the opposite end of the chamber. When each of these 32 subjects touched the prod, shock was administered through the wirewrapped prod, as in Experiment 1. Contact with the prod marked the beginning of the 15-min test session during which the rats' behavior was viewed on closed circuit television and the amount of time each rat spent moving bedding material toward the prod with snout and forepaws, that is, the duration of burying, was recorded on an event recorder. The height of the bedding at the base of the prod was measured following each test. The 32 noshock controls (16 one-compartment and 16 two-compartment rats) were not shocked when they touched the prod but were otherwise treated identically to the shock subjects.

\section{Results}

It is apparent from Figure 3 that both the duration of burying $[F(1,60)=42.11, p<.0001]$ and the height of the bedding material accumulated at the prod $[F(1,60)=22.39, p<.0001]$ were substantially greater for rats in the two groups receiving shock. Although the availability of a second compartment did not significantly reduce the height of the bedding accumulated at the prod [main effect, $F(1,60)=1.13$, $\mathrm{p}>.05$; interaction effect, $F(1,60)=.76, \mathrm{p}>.05]$, the reduction in the duration of burying produced by the availability of the second, safe compartment was reflected in a significant main effect $[F(1,60)$ $=8.34, p<.005]$ and a significant interaction effect $[F(1,60)=6.84, p<.01]$. However, even in the twocompartment condition, the shock rats buried longer $[\mathrm{t}(30)=3.80, \mathrm{p}<.002]$ and accumulated significantly higher piles of bedding at the prod $[\mathrm{t}(30)=3.04$, $\mathrm{p}<.006]$ than did their no-shock controls. The

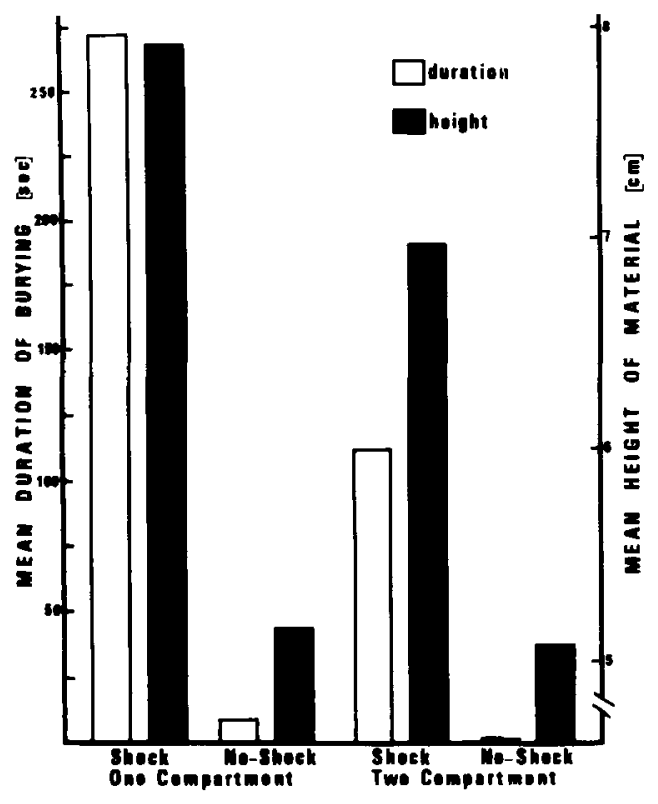

Figure 3. Mean duration of burying and mean height the bedding material accumulated at the prod by shock and no-shock subjects tested in a one- or two-compartment box. groups did not differ in their latency to contact the prod during the conditioning trial $[\mathrm{F}(3,60)=1.29$, $\mathrm{p}>.20]$.

\section{GENERAL DISCUSSION}

The purpose of the present experiments was to determine whether increasing the rat's capacity to flee from a well-defined shock source would decrease the amount of conditioned defensive burying. Because previous studies of conditioned defensive burying had all been conducted in small, one-compartment boxes, it was possible that conditioned defensive burying occurs only when rats are forced to remain in the immediate vicinity of the source of aversive stimulation.

Increasing the rats' opportunity for flight, either by increasing the size of the test chamber (Experiment 1) or by dividing the test chamber in two to create a second, safe compartment (Experiment 2), reduced the amount of defensive burying. In no instance, however, did a group of shocked subjects fail to bury significantly longer and accumulate significantly higher piles of bedding at the shock source than did its unshocked control group. Although the amount of conditioned defensive burying does appear to be influenced by the opportunity to perform competing defensive responses, it is not limited to situations in which rats are restricted to the immediate vicinity of the source of aversive stimulation.

The observation that conditioned defensive burying is not restricted to situations in which flight is impossible is consistent with the view (Hudson, 1950; Pinel \& Treit, 1978) that conditioned burying may have an important defensive function for rodents in their natural habitat, where there is usually an opportunity to flee from aversive stimuli. Although there is good evidence (e.g., Calhoun, 1962; Owings \& Coss, 1978) that burying can function as a defensive response for rodents in their natural habitat, it is usually not clear whether the objects of such behavior are innately determined or are established through conditioning. Owings and Coss (1978) provided evidence that the propensity of ground squirrels (Spermophilus beecheyi) to spray sand at snakes is innate; laboratory-reared ground squirrels sprayed sand at a snake, but not at a mechanical control object, on first encounter. Conversely, there have been no unambiguous reports of conditioned burying in wild rodents.

There have been two recent reports of conditioned defensive burying by laboratory rats in situations comparable in some ways to what a wild rodent might encounter in its natural environment (Terlecki et al., 1979; Wilkie, MacLennan, \& Pinel, 1979). Terlecki et al. found burying to be a major component of the rat's neophobic reaction to a mousetrap first encountered in a familiar environment. 
Rats previously habituated to the trap would bury it only if they had experienced its aversive effect. Although the rats reacted in the same way to a flashbulb presented in a familiar environment, a wooden, wire-wrapped prod or a length of polyethylene tubing was rarely the object of burying behavior unless it had been the source of electric shock or airblast, respectively. Rats seem to enter the experimental environment with an already established tendency to bury some novel objects (unconditioned defensive burying), and they readily learn to selectively bury a particular object that has been the source of any one of a variety of aversive stimuli (conditioned defensive burying). In the study by Wilkie et al., laboratory rats received standard taste aversion conditioning; as in previous experiments, rats were injected with lithium chloride following the consumption of a novel saccharin solution. This led to the usual decline in saccharin consumption; however, more important from the present perspective was the observation that every subject selectively buried the spout with bedding material from the floor of the chamber.

The present results also make an important practical point; the size and shape of test chambers can have a significant effect on the outcome of experiments using the conditioned defensive-burying paradigm. For example, in view of the simple inverse relationship between chamber size and burying (Ex- periment 1 ), it is now feasible to manipulate "baseline" levels of conditioned defensive burying by adjusting the size of the test chamber.

\section{REFERENCE NOTE}

1. Pinel, J. P. J., Treit, D., \& Wilkie, D. M. Constraints on avoidance learning: Burying an unfounded assumption. Paper presented at the Northeastern Regional meeting of the Animal Behavior Society, St. John's, Newfoundland, 1977.

\section{REFERENCES}

CAlhoun, J. B. The ecology and sociology of the Norway rat (U.S. Department of Health, Education \& Welfare). Washington, D.C: U.S. Government Printing Office, 1962.

Hudson, B. B. One-trial learning in the domestic rat. General Psychology Monographs, 1950, 41, 99-145.

Owings, D. H., \& Coss, R. G. Snake mobbing by California ground squirrels: Adaptive variation and ontogeny. Behaviour, $1978,62,50-69$.

Pinel, J. P. J., \& Treit, D. Burying as a defensive response in rats. Journal of Comparative and Physiological Psychology, 1978, 92, 708-712.

Terlecki, L. J., Pinel, J. P. J., \& Treit, D. Conditioned and unconditioned defensive burying in the rat. Learning and Motivation, 1979, 10, 337-350.

Wilkie, D. M., Maclennan, A. J., \& Pinel, J. P. J. Rat defensive behavior: Burying noxious food. Journal of the Experimental Analysis of Behavior, 1979, 31, 299-306.

(Received for publication August 6, 1979; revision accepted February 8,1980 .) 